\title{
Enhancement of shot noise due to the fluctuation of Coulomb interaction
}

\author{
Duo Li, Lei Zhang, Fuming Xu, and Jian Wang* \\ Department of Physics and the Center of Theoretical and Computational Physics, The University of Hong Kong, Hong Kong, China
}

(Received 1 November 2011; revised manuscript received 3 February 2012; published 2 April 2012)

\begin{abstract}
For a resonant tunneling structure, it was found experimentally that the shot noise is super-Poissonian in the negative differential region (NDR). From a semiclassical analysis, it is believed that the super-Poissonian behavior is due to fluctuation of the Coulomb interaction. Although there are many studies on shot noise in mesocopic or nanoscale systems, an accounting by first-principles quantum transport theory for super-Poissonian behavior in the NDR is still lacking. In this paper, we develop a theoretical formalism to investigate the contribution of fluctuations of the Coulomb interaction to the shot noise, based on the Keldysh nonequilibrium Green's function method. We applied our theory to study the behavior of dc shot noise of atomic junctions, using the method of nonequilibrium Green's functions combined with density functional theory (NEGF-DFT). In particular, for an atomic carbon wire consisting of four carbon atoms in contact with two $\mathrm{Al}(100)$ electrodes, a first-principles calculation within the NEGF-DFT formalism shows a NDR region in the $I-V$ curve at finite bias due to the effective band bottom of the Al lead. We calculated the shot noise spectrum using our theory. Our numerical result shows super-Poissonian behavior with a Fano factor larger than 1 in the NDR region, in agreement with the experimental result.
\end{abstract}

DOI: 10.1103/PhysRevB.85.165402

PACS number(s): 72.70.+m, 81.07.Nb, 73.63.-b

\section{INTRODUCTION}

Quantum effects have become remarkably significant in nanoscale semiconductors, and the traditional Boltzmann equation is no longer sufficient to describe transport phenomena. As proposed by Schottky in his seminal work, ${ }^{1}$ partition noise, or shot noise, results from the quantization of charge. Accordingly, when electrons are uncorrelated, the classical value of the Fano factor, which describes the magnitude of the electric fluctuation, should be 1 . When a Fano factor deviates from 1 , it is the signature of interactions between current flow in different probes. It is known that shot noise is influenced by two crucial factors, namely, the Pauli principle and the Coulomb interaction, which coexist in electronic systems. Specifically, the Pauli interaction can only suppress the Fano factor below 1, which corresponds to the sub-Poissonian case and has been confirmed convincingly by experiments. ${ }^{2-5}$ The Coulomb interaction, however, could either reduce the shot noise $^{6}$ or enhance it so that the Fano factor shows a superPoissonian value, ${ }^{7,8}$ depending on the details of the mesoscopic devices. Hence, quantum enhancement of shot noise from the classical value has been the subject of growing interest in recent years and is explored intensively. ${ }^{7-11}$ Recently, the quantum noise of cotunneling processes has been studied for quantum dot systems in the Coulomb blockade regime. ${ }^{12}$ A microscopic theory was developed for strong cotunneling processes. It was found that inelastic cotunneling can lead to super-Poissonian noise.

In mesoscopic systems shot noise is very important since it provides abundant information about transport properties of conductors, such as the kinetics of electrons, ${ }^{13}$ distributions of energy, ${ }^{14}$ and correlations of electronic wave functions. ${ }^{15}$ In addition, a shot noise enhancement in the negative differential resistance (NDR) region was observed experimentally, with or without a magnetic field, in a tunneling structure. ${ }^{7,8}$ Various mechanisms have been proposed to generate a NDR, including enhancement of tunnel barriers, ${ }^{16}$ strong intramolecular correlations, ${ }^{17}$ band-gap inducement, ${ }^{18}$ and so on. An early experiment by Li et al. suggested that, as the NDR region was approached, the suppressed value of the Fano factor would increase. ${ }^{5}$ Further exploration by Iannaccone et al. ${ }^{7}$ showed that, in the NDR region, the shot noise would go through a transition from a sub-Poissonian to a super-Poissonian value in a nonlinear fashion. Nevertheless, a NDR was not a sufficient condition to generate the enhancement: As Song et al. showed, there was no noise escalation in a superlattice tunnel diode even though its $I-V$ curve also exhibited a NDR region. ${ }^{19}$ This led to the conclusion that charge accumulation, related to the internal Coulomb potential, was ultimately responsible for the super-Poissonian shot noise. Given the good agreement between numerical calculations from semiclassical theory and those experiments, ${ }^{7}$ the Coulomb interaction was thought to be the reason for the noise enhancement. Since quantum effects dominate the transport behavior in mesoscopic systems, a quantum theory of shot noise capable of describing the enhancement in the NDR region is clearly needed. In 1999, Blanter and Büttiker studied the shot noise of a resonanttunneling quantum well theoretically using the scattering matrix method. ${ }^{20}$ In the nonlinear regime, Coulomb interaction (Hartree level) leads to hysteretic behavior in the $I-V$ curve. By including the fluctuations of the Coulomb interaction, they identified an important energy scale, termed the interaction energy, in the Fano factor. They found that in the NDR region where the interaction energy is very large, a superPoissonian behavior occurs due to the fluctuation of the Coulomb interaction.

Understanding the electronic transport properties of atomicwire-based structures is very important from the scientific viewpoint and due to its potential applications in molecular electronics. For example, by combining the LippmannSchwinger equation and density functional theory (DFT), a NDR in the tunneling regime of atomic carbon wires was predicted by Lang. ${ }^{21}$ The shot noise of silicon atomic wires has also been studied using the same approach. ${ }^{22}$ In this paper, we develop a general theory for dc shot noise by including the fluctuation of the Coulomb interaction. Our theory is based on 
the nonequilibrium Green's function (NEGF) method which can be coupled with DFT to study transport properties of nanodevices from first principles. As an application of our theory, we investigate the shot noise of an atomic carbon wire structure with four carbon atoms in the scattering region $\left(\mathrm{Al}-\mathrm{C}_{4}-\mathrm{Al}\right)$. Its $I-V$ characteristics and transport properties are well understood. ${ }^{23}$ It was found that a band-gap-induced NDR occurs at high bias due to a shift of conduction channels in the central region. We have used the traditional formula ${ }^{24,25}$

$$
\begin{aligned}
S_{\alpha \beta}= & (1 / 2)\left[\left\langle\Delta \hat{I}_{\alpha}(t) \Delta \hat{I}_{\beta}\left(t^{\prime}\right)\right\rangle+\left\langle\Delta \hat{I}_{\beta}\left(t^{\prime}\right) \Delta \hat{I}_{\alpha}(t)\right\rangle\right] \\
= & \frac{q^{2}}{\pi} \int d E\left\{\left[f_{\alpha}\left(1-f_{\alpha}\right)+f_{\beta}\left(1-f_{\beta}\right)\right] \operatorname{Tr}[\hat{T}]\right. \\
& \left.+\left(f_{\alpha}-f_{\beta}\right)^{2} \operatorname{Tr}[(1-\hat{T}) \hat{T}]\right\}
\end{aligned}
$$

to calculate the shot noise for the Al- $\mathrm{C}_{4}$-Al structure. Here $S_{\alpha \beta}$ represents the current correlation between leads $\alpha$ and $\beta, \hat{I}_{\alpha}$ is the current operator for current coming from lead $\alpha$ with $f_{\alpha}$ the Fermi distribution function in the corresponding lead, and the transmission coefficient $\hat{T}$ is given by $\Gamma_{\alpha} G^{r} \Gamma_{\beta} G^{a}$ with $G^{r, a}$ being the retarded and advanced Green's functions of the scattering region, respectively. In addition, $\Gamma_{\alpha, \beta}$ are the linewidth functions related to the coupling of the leads and the scattering region. Our results show that the shot noise is subPoissonian. When the fluctuation of the Coulomb interaction is included, large shot noise was found in the NDR region, showing super-Poissonian behavior.

Our paper is organized as follows. In Sec. II, we derive a general theory for dc shot noise when the fluctuation of the Coulomb interaction is included at first order. The detailed derivation is given in the Appendix. In Sec. III, we describe some technical details and show the numerical results in the atomic carbon chain system, along with an analysis and discussion of the results. Finally, a summary is given in Sec. IV.

\section{THEORETICAL FORMALISM}

In this section, a NEGF theory is developed to calculate dc shot noise in the regime of NDR, which involves the Coulomb interaction between electrons. A key ingredient of the new theory is that to account for large shot noise in the NDR region both the self-consistent Coulomb potential and its fluctuations have to be considered.

\section{A. General expression}

We start from a quantum coherent two-lead conductor defined by the Hamiltonian

$$
\begin{aligned}
\hat{H}_{0}= & \sum_{k \alpha} \epsilon_{k \alpha} \hat{C}_{k \alpha}^{\dagger} \hat{C}_{k \alpha}+\sum_{n}\left(\epsilon_{n}+U_{n}\right) d_{n}^{\dagger} d_{n} \\
& +\sum_{k \alpha n}\left[t_{k \alpha n} \hat{C}_{k \alpha}^{\dagger} \hat{d}_{n}+\text { c.c. }\right]
\end{aligned}
$$

where $\hat{C}_{k \alpha}^{\dagger}\left(\hat{C}_{k \alpha}\right)$ and $d_{n}^{\dagger}\left(d_{n}\right)$ are the creation (annihilation) operators of electrons in the leads and the scattering region, respectively. The first term describes leads on which dc voltages are applied, and $\epsilon_{k \alpha}=\epsilon_{k \alpha}^{(0)}+q v_{\alpha}$ where $\epsilon_{k \alpha}^{(0)}$ are the energy levels in the lead $\alpha$ and $v_{\alpha}$ stands for an external voltage. The second term is for the isolated central region, where the

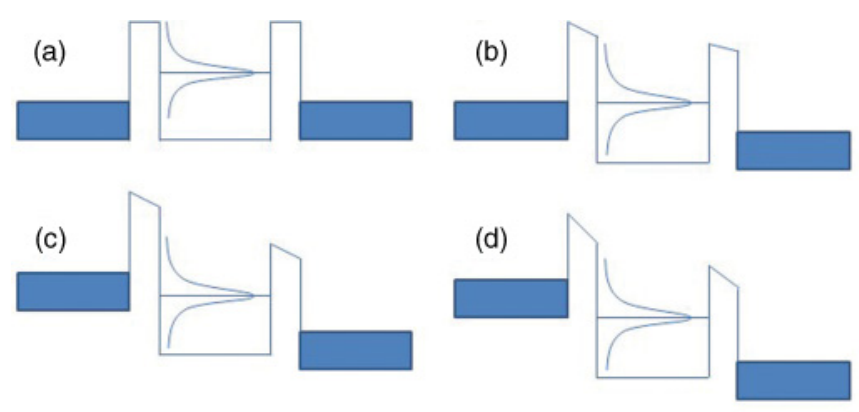

FIG. 1. (Color online) Graphical band profile for nanoscale devices in the presence of bias. At zero bias, the resonant level is above the Fermi level (a). As the bias increases, the Fermi level of the left lead increases and is above the resonant level (b) and (c), giving rise to a large increase of current. As the bias increases further, the resonant level is below the band bottom of the left lead (d), leading to a NDR.

self-consistent internal Coulomb potential under the Hartree approximation is defined as

$$
U_{n}=q \sum_{m} V_{n m}\left\langle d_{m}^{\dagger} d_{m}\right\rangle,
$$

where $V_{n m}$ is a matrix element of the Coulomb potential. In real space $V\left(x, x^{\prime}\right)=1 /\left|x-x^{\prime}\right| ; q$ is the electron charge. The last term corresponds to a coupling between the central region and leads described by a coupling constant $t_{k \alpha n}$.

The current operator of the lead $\alpha$ is defined as $(\hbar=1)$

$$
\hat{I}_{\alpha_{0}}(t)=q \frac{d \hat{N}_{\alpha}}{d t},
$$

where $\hat{N}_{\alpha}=\sum_{k} \hat{C}_{k \alpha}^{\dagger} \hat{C}_{k \alpha}$ is the number operator for electrons in the lead $\alpha$.

From the Heisenberg equation of motion

$$
\frac{d \hat{N}_{\alpha}}{d t}=-i\left[\hat{N}_{\alpha}, \hat{H}_{0}\right]
$$

we have

$$
\frac{d \hat{N}_{\alpha}}{d t}=-i \sum_{k n}\left[t_{k \alpha n} \hat{C}_{k \alpha}^{\dagger} \hat{d}_{n}\right]+\text { H.c. },
$$

where $\hat{H}$ is the system Hamiltonian with constant Coulomb potential and H.c. denotes the Hermitian conjugate. Hence the current operator becomes

$$
\hat{I}_{\alpha_{0}}(t)=-i q \sum_{k n}\left[t_{k \alpha n} \hat{C}_{k \alpha}^{\dagger}(t) \hat{d}_{n}(t)\right]+\text { H.c. }
$$

On the mean-field level, the current is a functional of the Coulomb interaction, i.e., $\hat{I}=\hat{I}[\langle\hat{U}\rangle]$. Here we have treated the operator of the Coulomb interaction $\hat{U}$ as a $C$ number, meaning that the fluctuation of the Coulomb interaction is assumed not important. However, this is not always true. For instance, in order to reflect the Coulomb interaction between electrons in the NDR region, we have to consider the fluctuation of the Coulomb potential. Figure 1 shows the physical picture of the NDR. For simplicity, we assume that the scattering region has one resonant level $E_{0}$ with a width characterizing the lifetime of the resonant level. We also 
assume that there is an effective band bottom for the lead, which is crucial for the phenomenon of NDR. As shown in Fig. 1, when the bias voltage is increased the current increases because the resonant level is brought down by the external bias. As the bias is increased further such that the resonant level falls below the band bottom of the lead, the current starts to decrease, giving rise to the NDR. The above physical picture is static, where the Coulomb potential is included on the mean-field level and the correlation effect of the Coulomb interaction has been neglected. For the current correlation in the NDR region, the correlation effect of the Coulomb interaction has to be considered. In this picture, when the resonant energy level $E_{0}$ is about to fall below the band bottom of the lead, the internal potential of the scattering region due to the Coulomb interaction of injected electrons will push it up, leading to a positive correlation between incoming electron flows. ${ }^{7}$ This positive correlation is a dynamic process and cannot be described by a Hartree field. In other words, the fluctuation of the Coulomb interaction has to be considered for a positive correlation in the NDR region. As demonstrated by Larade et $_{\text {al }}{ }^{23}$ and confirmed by our calculation, for an atomic wire with an even number of carbon atoms like $\mathrm{C}_{4}$ and $\mathrm{C}_{6}$, the coupling between the Al lead and the lowest unoccupied molecular orbital (LUMO) state of the even number of carbon atoms becomes very weak as the bias enters the NDR region. In other words, there is an effective band bottom responsible for the NDR. Therefore, the fluctuation of the Coulomb potential $\hat{U}$ should be important. For odd-number wires such as $C_{5}$ and $\mathrm{C}_{7}$, however, there is no apparent NDR effect. Hence there is no effective band bottom and the fluctuation of $\hat{U}$ can be neglected.

To treat the fluctuation of the Coulomb interaction, we follow the idea of Ref. 20 and expand the current in terms of the Coulomb potential operator about its equilibrium value up to linear order. After the expansion, the total current in real space can be expressed as

$$
\begin{aligned}
\hat{I}_{\alpha}(t) & \simeq \hat{I}_{\alpha_{0}}(t)+\left.\sum_{i} \frac{\delta \hat{I}_{\alpha_{0}}(t)}{\delta \hat{U}_{i}(t)}\right|_{\hat{U}_{i}(t)=U_{i}}\left[\hat{U}_{i}(t)-U_{i}\right] \\
& \simeq \hat{I}_{\alpha_{0}}(t)+\sum_{i} \frac{\delta I_{\alpha}}{\delta U_{i}}\left[\hat{U}_{i}(t)-U_{i}\right] \\
& =\hat{I}_{\alpha_{0}}(t)+\sum_{i} \lambda_{\alpha i} \delta \hat{U}_{i}(t)
\end{aligned}
$$

where $I_{\alpha}=\left\langle\hat{I}_{\alpha_{0}}\right\rangle, U_{i}=\left\langle\hat{U}_{i}\right\rangle$, and $\delta \hat{U}_{i}(t)=\hat{U}_{i}(t)-U_{i}$. We have also introduced the quantity $\lambda_{\alpha i}=\delta I_{\alpha} / \delta U_{i}$.

It is easy to see that this new current operator gives the same current but different shot noise. With the new current operator, the current correlation is obtained up to linear order in $\delta U$,

$$
\begin{aligned}
S_{\alpha \beta}^{(1)} & =\left\langle\Delta \hat{I}_{\alpha}(t) \Delta \hat{I}_{\beta}\left(t^{\prime}\right)\right\rangle=\left\langle\left(\hat{I}_{\alpha}(t) \hat{I}_{\beta}\left(t^{\prime}\right)\right\rangle-I_{\alpha} I_{\beta}\right. \\
& \simeq\left\langle\hat{I}_{\alpha_{0}}(t) \hat{I}_{\beta_{0}}\left(t^{\prime}\right)\right\rangle-I_{\alpha} I_{\beta}+\Delta_{\alpha \beta}^{(1)} .
\end{aligned}
$$

where $S_{\alpha \beta}=(1 / 2)\left[S_{\alpha \beta}^{(1)}+S_{\alpha \beta}^{(2)}\right]$. The first two terms in Eq. (9) correspond to the current correlation $S_{\alpha \beta 0}^{(1)}$ in the absence of Coulomb potential fluctuation. They have been calculated before and are given by Eq. (1). The last term in Eq. (9) is defined as

$$
\begin{aligned}
\Delta_{\alpha \beta}^{(1)}= & \sum_{i}\left[\lambda_{\beta i}\left\langle\hat{I}_{\alpha_{0}}(t) \hat{U}_{i}\left(t^{\prime}\right)\right\rangle+\lambda_{\alpha i}\left\langle\hat{U}_{i}(t) \hat{I}_{\beta_{0}}\left(t^{\prime}\right)\right\rangle\right. \\
& \left.-\left(\lambda_{\alpha i} U_{i} I_{\beta}+\lambda_{\beta i} U_{i} I_{\alpha}\right)\right] .
\end{aligned}
$$

Using the NEGF method, we have derived the expression for $\Delta_{\alpha \beta}^{(1)}$ (see the Appendix). Finally, the shot noise in the presence of Coulomb potential fluctuation is written as

$$
S_{\alpha \beta}=S_{\alpha \beta 0}+\Delta_{\alpha \beta},
$$

where

$$
\Delta_{\alpha \beta}=-\frac{q^{2}}{2 \pi} \sum_{i j}\left[\lambda_{\beta i} V_{i j} \operatorname{Im}\left(\Xi_{\alpha j j}\right)+\lambda_{\alpha i} V_{i j} \operatorname{Im}\left(\Xi_{\beta j j}\right)\right]
$$

with

$\lambda_{\alpha i}=-\frac{q}{2 \pi} \int d E \sum_{\beta}\left(f_{\alpha}-f_{\beta}\right)\left(G^{r} \Gamma_{\beta} G^{a} \Gamma_{\alpha} G^{r}\right)_{i i}+$ H.c.

and

$$
\begin{aligned}
\Xi_{\alpha}= & i \int d E\left[G^{r} \Gamma_{\alpha} G^{r} \Sigma^{>} G^{a} f_{\alpha}-G^{r} \Sigma^{<} G^{a} \Gamma_{\alpha} G^{a}\left(f_{\alpha}-1\right)\right. \\
& \left.+G^{r} \Sigma^{<} G^{a} \Gamma_{\alpha} G^{r} \Sigma^{>} G^{a}\right] .
\end{aligned}
$$

Equations (11)-(14) represent the central result of this paper. To calculate the shot noise, one has to solve the Green's function together with the Poisson equation,

$$
G^{r}=\frac{1}{E-H-U-V_{\mathrm{xc}}-\Sigma^{r}}
$$

and

$$
\nabla^{2} U(x)=4 \pi i q \int \frac{d E}{2 \pi} G^{<}(E, U, x, x),
$$

where $V_{\mathrm{xc}}$ is the potential due to the exchange and correlation effect in the first-principles calculation.

For a two-terminal device at zero temperature, we set $\alpha=L, \beta=R$, and $v_{R}>v_{L}=0$ so that $f_{L}\left(f_{L}-1\right)=0$ and $f_{R}\left(f_{L}-1\right)=0$ at zero temperature. We then obtain

$$
\begin{aligned}
\Xi_{L}= & i \int d E\left[G^{r} \Gamma_{L} G^{r} \Sigma^{>} G^{a} f_{L}\right. \\
& \left.-G^{r} \Sigma^{<} G^{a} \Gamma_{L} G^{a}\left(f_{L}-1\right)+G^{r} \Sigma^{<} G^{a} \Gamma_{L} G^{r} \Sigma^{>} G^{a}\right] \\
= & \int_{E_{F}-q v_{R}}^{E_{F}} d E\left(G^{r} \Gamma_{L} G^{r} \Gamma_{R} G^{a}+i G^{r} \Gamma_{L} G^{a} \Gamma_{L} G^{r} \Gamma_{R} G^{a}\right) .
\end{aligned}
$$

Similarly, we have

$$
\Xi_{R}=\int_{E_{F}-q v_{R}}^{E_{F}} d E\left(G^{r} \Gamma_{R} G^{r} \Gamma_{L} G^{a}+i G^{r} \Gamma_{R} G^{a} \Gamma_{R} G^{r} \Gamma_{L} G^{a}\right) .
$$

In addition, from Eq. (13), we have

$$
\begin{aligned}
& \lambda_{L}=-\frac{q}{2 \pi} \int_{E_{F}-q v_{R}}^{E_{F}} d E\left(G^{r} \Gamma_{R} G^{a} \Gamma_{L} G^{r}\right)+\text { H.c., } \\
& \lambda_{R}=\frac{q}{2 \pi} \int_{E_{F}-q v_{R}}^{E_{F}} d E\left(G^{r} \Gamma_{L} G^{a} \Gamma_{R} G^{r}\right)+\text { H.c. }
\end{aligned}
$$

With Eqs.(17), (18), and (19), the two-probe shot noise can be calculated. 


\section{NUMERICAL RESULTS}

In this section we use the state-of-the-art first-principles quantum transport package MATDCAL to investigate the general transport properties of atomic carbon chain systems coupled with Al leads. In the package a DFT calculation is carried out within the formalism of the Keldysh nonequilibrium Green's function method. Numerically, the effective Kohn-Sham (KS) equations are solved by a linear combination of atomic orbitals (LCAO) basis set. We define the atomic core by a nonlocal norm-conserving pseudopotential and treat the exchange and correlation at the level of the local density approximation (LDA). The DFT determines the atomic structure and the system Hamiltonian while the NEGF formalism contributes to the nonequilibrium transport properties. Under an external bias the transport boundary conditions are treated by real-space numerical techniques. For further reference, the theoretical background and the practical execution of this formalism can be found in Ref. 26. The numerical error tolerance is set to be $10^{-4}$ to confirm self-consistency.

Generally speaking, we have carried out our calculation on the atomic chain structure with four carbon atoms $\mathrm{Al}-\mathrm{C}_{4}-\mathrm{Al} .^{23}$ The carbon chain lies in the central simulation box in contact with electron reservoirs through two semi-infinite $\mathrm{Al}$ electrodes. The schematic structure is shown in Fig. 2 where there are $18 \mathrm{Al}$ atoms in the unit cell of the semi-infinite electrodes with a cross section along the (100) direction. The contact distance between the Al electrode and the carbon chain is fixed at 0.378 a.u. while the distance between neighboring carbon atoms is equal to 2.50 a.u. In our calculation, we have set the temperature to be zero.

Technically, the correction term in Eq. (12) has to be solved in real space due to the Coulomb-like interaction involving $V_{i j}$, which reads $V\left(x, x^{\prime}\right)=1 /\left|x-x^{\prime}\right|$ in real space. Since the quantities $\lambda$ and $\Xi$ play the role of charge, we can define the potential induced by $\Xi$,

$$
\Omega_{\alpha x}=\sum_{x^{\prime}} V\left(x, x^{\prime}\right) \operatorname{Im}\left[\Xi_{\alpha}\left(x^{\prime}, x^{\prime}\right)\right] .
$$

Since $\nabla_{x}^{2} V\left(x, x^{\prime}\right)=-4 \pi \delta\left(x-x^{\prime}\right), \Omega_{\alpha}$ satisfies the Poissonlike equation

$$
\nabla^{2} \Omega_{\alpha}(x)=-4 \pi \operatorname{Im}\left[\Xi_{\alpha}(x, x)\right]
$$

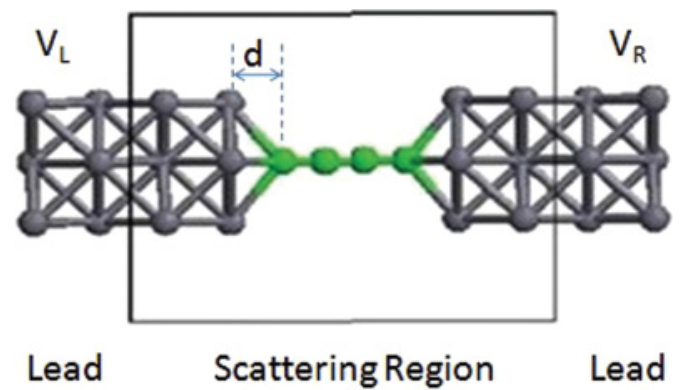

FIG. 2. (Color online) A schematic plot of the $\mathrm{Al}-\mathrm{C}_{4}-\mathrm{Al}$ system. An atomic wire with four carbon atoms is linked by two semi-infinite $\mathrm{Al}$ electrodes. The (100)-direction $\mathrm{Al}$ electrodes extend to $\pm \infty$ where the electron reservoirs are located.

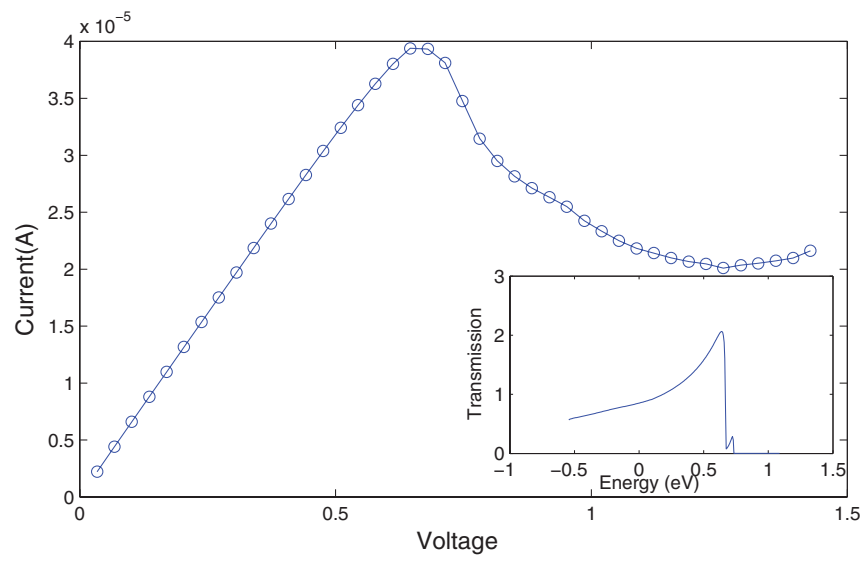

FIG. 3. (Color online) The $I-V$ curve of $\mathrm{Al}-\mathrm{C}_{4}-\mathrm{Al}$ structure. A NDR region begins to show up around $0.65 \mathrm{~V}$. Inset: the transmission coefficient for this system at zero bias.

We solve this equation for the leads to obtain the boundary condition for the scattering region. It turns out that $\Omega_{\alpha}(x)$ in the lead is very small so that the boundary condition of $\Omega_{\alpha}(x)$ can be safely set to zero. Once $\Omega_{\alpha}(x)$ is obtained the correction term can be easily calculated from Eq. (12):

$$
\Delta_{\alpha \beta}=-\frac{q^{2}}{2 \pi} \int\left[\lambda_{\beta}(x) \Omega_{\alpha}(x)+\lambda_{\alpha}(x) \Omega_{\beta}(x)\right] d x .
$$

The $I-V$ characteristic is shown in Fig. 3, where the inset shows the transmission coefficient $T$ versus the energy $E$ at zero bias. The shot noise and the corresponding Fano factor are shown in Fig. 4. In Figs. 3 and 4, the Coulomb interaction is included on the Hartree level and the Coulomb potential fluctuation is neglected. The following observations are in order. (1) The $I-V$ curve is similar to the result obtained by Larade et al. $^{23}$ (2) At zero bias the resonant energy is higher than the Fermi energy of the system (chosen to be zero). As we apply a voltage to the right electrode, the resonant energy level should drop and move closer to the Fermi energy of the left electrode. With an increase of voltage, the effective band bottom of the emitter and the main resonant level will be aligned, and this gives the maximum current around $0.65 \mathrm{~V}$. When the voltage increases further, a significant decrease of current occurs. (3) The shot noise in the absence of Coulomb potential fluctuation

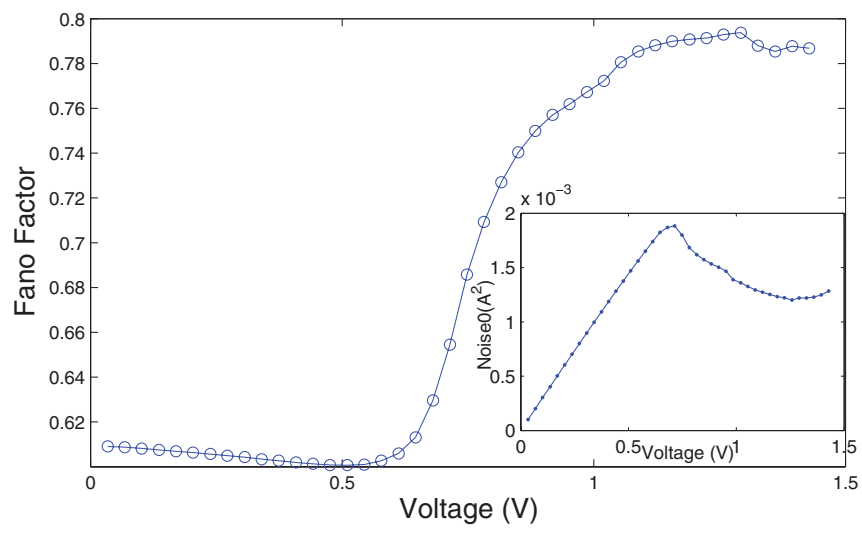

FIG. 4. (Color online) The Fano factor derived from the unmodified model which shows sub-Poissonian behavior. Inset: the noise spectrum calculated by Eq. (1). 


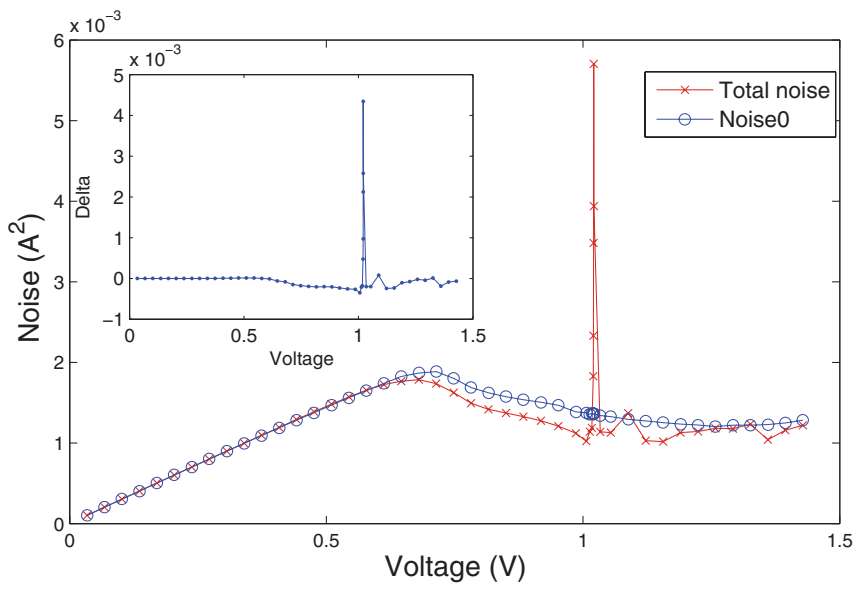

FIG. 5. (Color online) The noise spectrum plotted as a function of the applied voltage at $0 \mathrm{~K}$. Inset: the correction term for a two-terminal system.

has a similar behavior to that of the $I-V$ curve except that the maximum is at $0.7 \mathrm{~V}$ instead of $0.65 \mathrm{~V}$. (4) The Fano factor is nearly a constant of the order of 0.6 in the positive differential resistance (PDR) region when the bias is smaller than $0.6 \mathrm{~V}$. It starts to increase sharply upon entering the NDR region and the Fano factor eventually shoots up to 0.8 . We conclude that, although the Fano factor calculated on the Hartree level shows enhancement in the NDR region, it is still sub-Poissonian, which does not agree with the experimental result. ${ }^{7}$

In general, when electrons tunnel through the left barrier to occupy empty energy levels, the Pauli principle inhibits other tunneling electrons from reaching the same energy level; they go into higher ones. As a consequence the Pauli exclusion principle gives a negative current correlation. The Coulomb interaction, however, can give either a positive or a negative correlation effect to shot noise. This can be understood as follows. It is known that the maximum current corresponds to the situation that the energy of incoming electron is in line with the resonant level. Hence, in both the PDR and NDR regions, most electrons are off resonance. Due to the Coulomb interaction the incoming electron can push up the resonant level so that the next electron will be further away from the resonance in the PDR region or closer to the resonance in the NDR region, giving rise negative or positive correlations. Our numerical results indeed confirm this physical picture. In Figs. 5 and 6 we present the results for the shot noise and Fano factor in the presence of fluctuations of the Coulomb interaction. We have also included the shot noise and Fano factor in the absence of Coulomb potential fluctuation for comparison. In Fig. 5, we see that the correction term solved via the Poisson-like equation is very small at low bias when $V<0.5 \mathrm{~V}$ and becomes negative until around $1.0 \mathrm{~V}$ where the shot noise increases sharply. In Fig. 6, we see that a large Fano factor great than 3 occurs near $V=1.0 \mathrm{~V}$. This result is in qualitative agreement with others' work. $7,20,27$

\section{CONCLUSION}

The traditional formalism can only describe the suppression of shot noise, which corresponds to the PDR region. In order to treat enhancement of shot noise correctly in the NDR region,

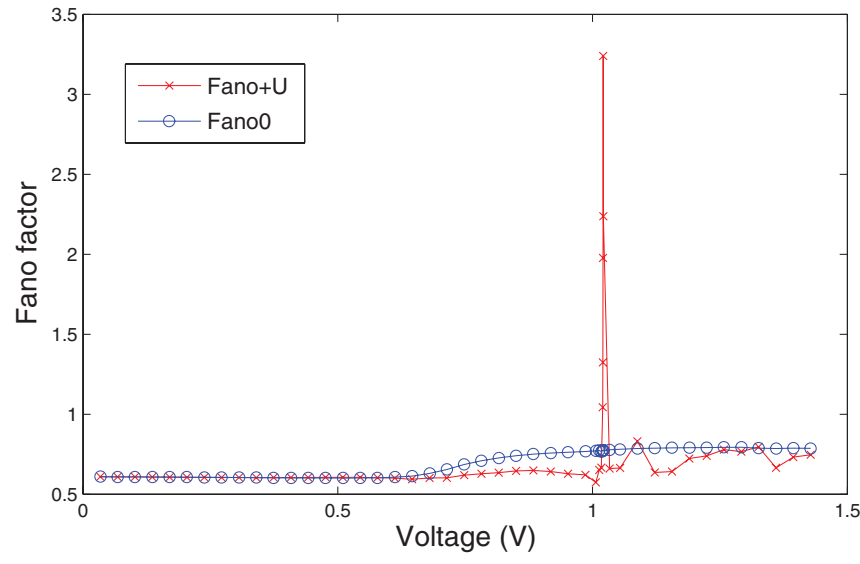

FIG. 6. (Color online) The Fano factor plotted as a function of the applied voltage for the $\mathrm{Al}-\mathrm{C}_{4}-\mathrm{Al}$ system at $0 \mathrm{~K}$. Circles: the Fano factor calculated by Eq. (1) for the same system.

we have to include the fluctuation of the Coulomb interaction. In this paper we have developed such a general dc theory for calculating the shot noise in the NDR region. The theoretical framework is based on a combination of the NEGF-DFT formalism with the self-consistent Coulomb potential and its fluctuation included. Our theory [Eq. (11)] can also be applied to mesoscopic conductors. We have applied our theory to molecular devices. Specifically, we have calculated the shot noise of an $\mathrm{Al}-\mathrm{C}_{4}-\mathrm{Al}$ structure, which is an ideal system since its $I-V$ curve exhibits a NDR region. We found a large Fano factor in the NDR region, exhibiting super-Poissonian behavior.

\section{ACKNOWLEDGMENTS}

We thank Dr. Y. X. Xing for checking all the algebra in the theory part of the paper. This work was financially supported by the Research Grant Council (Grant No. HKU 705409P) and the University Grant Council (Contract No. AoE/P-04/08) of the Government of HKSAR.

\section{APPENDIX}

In this Appendix, we will derive the expressions for $\lambda_{\alpha}$ and $\Delta_{\alpha \beta}$ given in Eqs. (12) and (13) using the theory of the NEGF.

\section{Expression for $\lambda_{\alpha}$}

Using the NEGF, the current is given by

$$
I_{\alpha}=-\frac{q}{2 \pi} \int d E \sum_{\beta}\left[\operatorname{Tr}\left(\Gamma_{\alpha} G^{r} \Gamma_{\beta} G^{a}\right)\right]\left(f_{\alpha}-f_{\beta}\right),
$$

where $f_{\alpha, \beta}$ are the Fermi distribution functions in the corresponding leads, $G^{r, a}$ are the respective retarded and advanced Green functions of the scattering region, and $\Gamma_{\alpha, \beta}$ are the linewidth functions related to the coupling of leads and the scattering region.

To obtain $\lambda_{\alpha}$, we first calculate the following quantity:

$$
\lambda_{\alpha i}(E)=-\frac{q}{2 \pi} \sum_{\beta} \operatorname{Tr} \frac{\partial}{\partial U_{i}}\left[\Gamma_{\alpha} G^{r} \Gamma_{\beta} G^{a}\right]
$$




$$
\begin{aligned}
& =-\frac{q}{2 \pi} \sum_{\beta} \operatorname{Tr}\left[\Gamma_{\alpha}\left(\frac{\partial G^{r}}{\partial U_{i}}\right) \Gamma_{\beta} G^{a}+\Gamma_{\alpha} G^{r} \Gamma_{\beta}\left(\frac{\partial G^{a}}{\partial U_{i}}\right)\right] \\
& =-\frac{q}{2 \pi} \sum_{\beta} \operatorname{Tr}\left[\Gamma_{\alpha}\left(\frac{\partial G^{r}}{\partial U_{i}}\right) \Gamma_{\beta} G^{a}\right]+\text { H.c. }
\end{aligned}
$$

The quantity $\partial_{U} G^{r}$ can be calculated from the Dyson equation $^{28}$

$$
G^{r}=G_{0}^{r}+G_{0}^{r} U G^{r}
$$

where $G_{0}^{r}$ is the retarded Green's function in the absence of Coulomb interaction. Taking the derivative with respect to $U_{i}$, we have

$$
\frac{\partial}{\partial U_{i}} G^{r}=\frac{\partial}{\partial U_{i}}\left(G_{0}^{r} U G^{r}\right)=G_{0}^{r} D_{i} G^{r}+G_{0}^{r} U \frac{\partial G^{r}}{\partial U_{i}},
$$

where $D_{i}$ is a diagonal matrix with the matrix element $\left(D_{i}\right)_{j k}=\delta_{j i} \delta_{k i}$, i.e., there is only one nonzero matrix element. From Eq. (A4), we find

$$
\frac{\partial}{\partial U_{i}} G^{r}=\frac{1}{1-G_{0}^{r} U} G_{0}^{r} D_{i} G^{r}=G^{r} D_{i} G^{r},
$$

where we have used the following Dyson equation again:

$$
G^{r}=\frac{1}{1-G_{0}^{r} U} G_{0}^{r} .
$$

Substituting Eq. (A5) into Eq. (A2), we get

$$
\begin{aligned}
\lambda_{\alpha i}(E) & =-\frac{q}{2 \pi} \sum_{\beta} \operatorname{Tr}\left(\Gamma_{\alpha} G^{r} D_{i} G^{r} \Gamma_{\beta} G^{a}\right)+\text { H.c. } \\
& =-\frac{q}{2 \pi} \sum_{\beta} \operatorname{Tr}\left(G^{r} \Gamma_{\beta} G^{a} \Gamma_{\alpha} G^{r} D_{i}\right)+\text { H.c. } \\
& =-\frac{q}{2 \pi} \sum_{\beta}\left(G^{r} \Gamma_{\beta} G^{a} \Gamma_{\alpha} G^{r}\right)_{i i}+\text { H.c., }
\end{aligned}
$$

which is equivalent to Eq. (13).

\section{Expression for $\Delta_{\alpha \beta}$}

For simplicity, we deal explicitly with only the first term of $\Delta_{\alpha \beta}$ in Eq. (10), i.e., $\left\langle\hat{I}_{\alpha_{0}}(t) \hat{U}_{i}\left(t^{\prime}\right)\right\rangle$. The second term can be calculated similarly. Using the current and Coulomb potential operators in Eqs. (7) and (3), we obtain

$$
\begin{aligned}
& \left\langle\hat{I}_{\alpha_{0}}(t) \hat{U}_{i}\left(t^{\prime}\right)\right\rangle \\
& =\left\langle-i q^{2} \sum_{k n}\left[t_{k \alpha n} \hat{C}_{k \alpha}^{\dagger}(t) \hat{d}_{n}(t)-\text { H.c. }\right] \sum_{m} V_{i m} \hat{d}_{m}^{\dagger}\left(t^{\prime}\right) \hat{d}_{m}\left(t^{\prime}\right)\right\rangle \\
& =-i q^{2} \sum_{k n m} V_{i m}\left[t_{k \alpha n}\left\langle\hat{C}_{k \alpha}^{\dagger}(t) \hat{d}_{n}(t) \hat{d}_{m}^{\dagger}\left(t^{\prime}\right) \hat{d}_{m}\left(t^{\prime}\right)\right\rangle\right. \\
& \left.\quad-t_{k \alpha n}^{*}\left\langle\hat{d}_{n}^{\dagger}(t) \hat{C}_{k \alpha}(t) \hat{d}_{m}^{\dagger}\left(t^{\prime}\right) \hat{d}_{m}\left(t^{\prime}\right)\right\rangle\right] \\
& =-i q^{2} \sum_{k n m} V_{i m}\left[t_{k \alpha n}\left\langle\hat{C}_{k \alpha}^{\dagger}(t) \hat{d}_{m}\left(t^{\prime}\right)\right\rangle\left\langle\hat{d}_{n}(t) \hat{d}_{m}^{\dagger}\left(t^{\prime}\right)\right\rangle\right. \\
& \left.-t_{k \alpha n}^{*}\left\langle\hat{d}_{n}^{\dagger}(t) \hat{d}_{m}\left(t^{\prime}\right)\right\rangle\left\langle\hat{C}_{k \alpha}(t) \hat{d}_{m}^{\dagger}\left(t^{\prime}\right)\right\rangle\right]+I_{\alpha} U_{i},
\end{aligned}
$$

where $I_{\alpha}=-i q \sum_{k n} t_{k \alpha n}\left\langle\hat{C}_{k \alpha}^{\dagger}(t) \hat{d}_{n}(t)\right\rangle+$ c.c. and $U_{i}=$ $q \sum_{m} V_{i m}\left\langle\hat{d}_{m}^{\dagger}\left(t^{\prime}\right) \hat{d}_{m}\left(t^{\prime}\right)\right\rangle$.
In terms of Green's functions ${ }^{29}$

$$
\begin{aligned}
G_{m, k \alpha}^{<}\left(t, t^{\prime}\right) & =i\left\langle\hat{C}_{k \alpha}^{\dagger}\left(t^{\prime}\right) \hat{d}_{m}(t)\right\rangle, \\
G_{n m}^{>}\left(t^{\prime}, t\right) & =-i\left\langle\hat{d}_{n}\left(t^{\prime}\right) \hat{d}_{m}^{\dagger}(t)\right\rangle, \\
G_{m n}^{<}\left(t, t^{\prime}\right) & =i\left\langle\hat{d}_{n}^{\dagger}\left(t^{\prime}\right) \hat{d}_{m}(t)\right\rangle \\
G_{k \alpha, m}^{>}\left(t^{\prime}, t\right) & =-i\left\langle\hat{C}_{k \alpha}\left(t^{\prime}\right) \hat{d}_{m}^{\dagger}(t)\right\rangle,
\end{aligned}
$$

we obtain $\left\langle\hat{I}_{\alpha_{0}}(t) \hat{U}_{i}\left(t^{\prime}\right)\right\rangle$

$$
\begin{aligned}
& =-i q^{2} \sum_{k n m} V_{i m}\left[t_{k \alpha n} G_{m, k \alpha}^{<}\left(t, t^{\prime}\right) G_{n m}^{>}\left(t^{\prime}, t\right)\right. \\
& \left.-t_{k \alpha n}^{*} G_{m n}^{<}\left(t, t^{\prime}\right) G_{k \alpha, m}^{>}\left(t^{\prime}, t\right)\right]+I_{\alpha} U_{i} .
\end{aligned}
$$

Applying the Langreth theorem of analytic continuation ${ }^{30}$ and suppressing time indices, we have

$$
\begin{aligned}
& G_{m, k \alpha}^{<}=\sum_{l}\left(G_{m l}^{r} t_{k \alpha l}^{*} g_{k \alpha}^{<}+G_{m l}^{<} t_{k \alpha l}^{*} g_{k \alpha}^{a}\right), \\
& G_{k \alpha, m}^{>}=\sum_{l}\left(g_{k \alpha}^{>} t_{k \alpha l} G_{l m}^{a}+g_{k \alpha}^{r} t_{k \alpha l} G_{l m}^{>}\right),
\end{aligned}
$$

where $g_{k \alpha}^{\langle,\rangle, r, a}$ are the corresponding Green's functions in the lead $\alpha$.

For dc transport, the Green's functions depend only on $t^{\prime}-t$. After a Fourier transform from time to energy, we obtain

$$
\begin{aligned}
& \left\langle\hat{I}_{\alpha_{0}} \hat{U}_{i}\right\rangle-I_{\alpha} U_{i} \\
& =-\frac{i q^{2}}{2 \pi} \sum_{k n m l} V_{i m} \int d E\left[t _ { k \alpha n } \left(G_{m l}^{r} t_{k \alpha l}^{*} g_{k \alpha}^{<}\right.\right. \\
& \left.+G_{m l}^{<} t_{k \alpha l}^{*} g_{k \alpha}^{a}\right) G_{n m}^{>}-t_{k \alpha n}^{*} G_{m n}^{<}\left(g_{k \alpha}^{>} t_{k \alpha l} G_{l m}^{a}\right. \\
& \left.\left.+g_{k \alpha}^{r} t_{k \alpha l} G_{l m}^{>}\right)\right] \\
& =-\frac{i q^{2}}{2 \pi} \sum_{m} V_{i m} \int d E\left[\left(G^{r} \Sigma_{\alpha}^{<}+G^{<} \Sigma_{\alpha}^{a}\right) G^{>}\right. \\
& \left.-G^{<}\left(\Sigma_{\alpha}^{>} G^{a}+\Sigma_{\alpha}^{r} G^{>}\right)\right]_{m m}=-\frac{i q^{2}}{2 \pi} \sum_{m} V_{i m}\left[\Xi_{\alpha}\right]_{m m},
\end{aligned}
$$

where $\Sigma_{\alpha}^{\langle,\rangle, r, a}$ are the corresponding functions of the selfenergy due to the lead $\alpha$. Using the Keldysh equation and properties of the Green's functions, ${ }^{28}$

$$
\begin{aligned}
G^{<} & =G^{r} \Sigma^{<} G^{a}, \\
\Sigma_{\alpha}^{a}(E)-\Sigma_{\alpha}^{r}(E) & =i \Gamma_{\alpha}\left(E-q v_{\alpha}\right), \\
\Sigma_{\alpha}^{<}(E) & =i \Gamma_{\alpha}\left(E-q v_{\alpha}\right) f_{\alpha}(E), \\
\Sigma_{\alpha}^{>}(E) & =i \Gamma_{\alpha}\left(E-q v_{\alpha}\right)\left(f_{\alpha}(E)-1\right),
\end{aligned}
$$

$$
\begin{aligned}
& \text { we find } \\
& \Xi_{\alpha}=i \int d E\left[G^{r} \Gamma_{\alpha} G^{r} \Sigma^{>} G^{a} f_{\alpha}-G^{r} \Sigma^{<} G^{a} \Gamma_{\alpha} G^{a}\left(f_{\alpha}-1\right)\right.
\end{aligned}
$$$$
\left.+G^{r} \Sigma^{<} G^{a} \Gamma_{\alpha} G^{r} \Sigma^{>} G^{a}\right] .
$$

Substituting this equation into Eqs. (A11) and (10), we finally arrive at

$$
\Delta_{\alpha \beta}^{(1)}=-\frac{i q^{2}}{2 \pi} \sum_{i j}\left(\lambda_{\beta i} V_{i j} \Xi_{\alpha j}-\lambda_{\alpha i} V_{i j} \Xi_{\beta j}^{*}\right),
$$

where we have used the fact that $\left\langle\hat{I}_{\alpha_{0}}(t) \hat{U}_{i}\left(t^{\prime}\right)\right\rangle=$ $\left\langle\hat{U}_{i}(t) \hat{I}_{\alpha_{0}}\left(t^{\prime}\right)\right\rangle^{\dagger}$. 
*jianwang@hkusua.hku.hk

${ }^{1}$ W. Schottky, Ann. Phys. (Leipzig) 57, 541 (1918).

${ }^{2}$ M. Henny, S. Oberholzer, C. Strunk, and C. Schonenberger, Phys. Rev. B 59, 2871 (1999).

${ }^{3}$ M. Reznikov, M. Heiblum, H. Shtrikman, and D. Mahalu, Phys. Rev. Lett. 75, 3340 (1995).

${ }^{4}$ A. Kumar, L. Saminadayar, D. C. Glattli, Y. Jin, and B. Etienne, Phys. Rev. Lett. 76, 2778 (1996).

${ }^{5}$ Y. P. Li, D. C. Tsui, J. J. Heremans, J. A. Simmons, and G. W. Weimann, Appl. Phys. Lett. 57, 774 (1990).

${ }^{6}$ T. Gonzalez, C. Gonzalez, J. Mateos, D. Pardo, L. Reggiani, O. M. Bulashenko, and J. M. Rubi, Phys. Rev. Lett. 80, 2901 (1998);

C. W. J. Beenakker, ibid. 82, 2761 (1999).

${ }^{7}$ G. Iannaccone, G. Lombardi, M. Macucci, and B. Pellegrini, Phys. Rev. Lett. 80, 1054 (1998).

${ }^{8}$ V. V. Kuznetsov, E. E. Mendez, J. D. Bruno, and J. T. Pham, Phys. Rev. B 58, R10159 (1998).

${ }^{9}$ E. R. Brown, IEEE Trans. Electron Devices 39, 2686 (1992).

${ }^{10}$ R. C. Liu, B. Odom, Y. Yamamoto, and S. Tarucha, Nature (London) 391, 263 (1998).

${ }^{11}$ Y. M. Blanter and M. Büttiker, Phys. Rep. 336, 1 (2000).

${ }^{12}$ D. Loss and E. V. Sukhorukov, Phys. Rev. Lett. 84, 1035 (2000);

E. V. Sukhorukov, G. Burkard, and D. Loss, Phys. Rev. B 63, 125315 (2001).

${ }^{13}$ R. Landauer, Nature (London) 392, 659 (1998).

${ }^{14}$ O. M. Bulashenko, J. M. Rubi, and V. A. Kochelap, Phys. Rev. B 62, 8184 (2000).
${ }^{15}$ T. Gramespacher and M. Büttiker, Phys. Rev. Lett. 81, 2763 (1998).

${ }^{16}$ N. Simonian, J. B. Li, and K. Likharev, Nanotechnology 18, 424006 (2007).

${ }^{17}$ Y. B. Kudasov, Bull. Russ. Acad. Sci.: Phys. 72, 172 (2008).

${ }^{18}$ T. Rakshit, G. C. Liang, A. W. Ghosh, and Supriyo Datta, Nano Lett. 4, 10 (2004).

${ }^{19}$ W. Song, E. E. Mendez, V. Kuznetsov, and B. Nielson, Appl. Phys. Lett. 82, 1568 (2003).

${ }^{20}$ Y. M. Blanter and M. Büttiker, Phys. Rev. B 59, 10217 (1999).

${ }^{21}$ N. D. Lang, Phys. Rev. B 55, 9364 (1997); N. D. Lang and Ph. Avouris, Phys. Rev. Lett. 84, 358 (2000).

${ }^{22}$ Y. C. Chen and M. Di Ventra, Phys. Rev. B 67, 153304 (2003).

${ }^{23}$ B. Larade, J. Taylor, H. Mehrez, and H. Guo, Phys. Rev. B 64, 075420 (2001).

${ }^{24}$ M. Buittiker, Phys. Rev. B 46, 485 (1992).

${ }^{25}$ Y. Wei, B. Wang, J. Wang, and H. Guo, Phys. Rev. B 60, 16900 (1999).

${ }^{26}$ J. Taylor, H. Guo, and J. Wang, Phys. Rev. B 63, 245407 (2001); 63, 121104 (2001).

${ }^{27}$ V. N. Do, P. Dollfus, and V. L. Nguyen, J. Appl. Phys. 100, 093705 (2006).

${ }^{28} \mathrm{H}$. Haug and A. Jauho, Quantum Kinetics in Transport and Optics of Semiconductors (Springer, Berlin, 1998), Chap. 12.

${ }^{29}$ S. Datta, Electronic Transport in Mesoscopic Systems (Cambridge University Press, Cambridge, 1995), Chap. 8.

${ }^{30}$ D. C. Langreth, in Linear and Nonlinear Electron Transport in Solids, edited by J. T. Devreese and E. Van Doren (Plenum, New York, 1976). 